- Short communication

\title{
A preliminary list of the migratory birds of the Padma charland, Rajshahi
}

\author{
A. M. Saleh Reza and Selina Parween ${ }^{*}$ \\ Department of Zoology, University of Rajshahi, Rajshahi-6205, Bangladesh
}

Key words: Migratory waterbirds, Padma charland, Rajshahi.

The mighty river Padma has become compartmentalized by the development of sandbars commonly called as 'charlands'. Charlands are generally a seasonal natural process of the riverine ecology, especially at the lower reach of the rivers. Sandbars or the 'char' is typically composed of sand, silt and/or small pebbles, which emerge as islands within the river channel or as attached land to the riverbanks. Padma shows meandering characteristic and produce stable attached char to the riverbanks and the entire process of char formation would be completed within twelve to fifteen years (Rahman \& Rahman, 2012) after 12-15 years it is turned into stable char. Stable chars are more resourceful than semi stable char (Sarker et al., 2003). Construction of large dams on the rivers, very often affect the flow and current of the river water, resulting in the formation of sandbars. The Padma river is known for heavy bank erosion, shifting channels, and sandbars that continually emerge in its course (Rahman \& Rahman, 2010).

There are a large number of charlands, small and large, have been formed in the Padma river which are quite high from the water level. On the part of the river that flowing adjacent to the Rajshahi district, there are few charlands which become continuous escaping at some parts and are quite old. During monsoon greater part of this charland becomes flooded. The chars at some part may be sandy, in some parts may be alluvial, having number of water pockets or channels of different size, and have a complex ecosystem. The Padma charland is mostly sandy, with parts having sand and alluvial mixed soil. On the areas having mixed soil the natural flora had grown and people use the land for agriculture because of its rich fertile soil. There are several villages with school and community health centre at the charland of Padma lying within the Rajshahi district. At the human habitation there are a number of species of trees and plants which produce fruits and woods. Besides that, plantation programs have been carried out to protect the river side from water current. The Padma charland has a great economic value by producing huge amount of sand, different types of crops, fruits and vegetables, riverine species of fish and fire woods.

Riverine habitats (water channels and sandbars) support a wide variety of bird species, many of them nests on sandbars, others forage along or in the water channel (Claassen,

\footnotetext{
*Corresponding author, e-mail: parween_s@yahoo.com
} 
2004). During winter season a number of migratory birds invades the Padma charland, and some people hunts these birds to earn money. The Padma charland inhabited by wildlife species, but there is no information on the wildlife of this important habitat. However, the riverine fish species were recorded by several workers, but a few reports have been published (Islam \& Hossain, 1983; Ali et al., 2013). Khan (1973) published a checklist of birds of Rajshahi, but did not mention the riverine species.

The present work is the result of seven years bird of watching at charlands of Padma extending from Premtali of Godagari Upazilla to Charghat Upazilla of the Rajshahi district, and here reported the list of migratory birds of the charland.

Study area and tenure: The study area extending from Premtali of Godagari Upazilla $\left(24^{\circ} 28^{\prime} \mathrm{N} 88^{\circ} 19.8^{\prime} \mathrm{E}\right)$ to Rajshahi Cadet College, Sardah of Charghat Upazilla $\left(24^{\circ} 17^{\prime} \mathrm{N}\right.$ $\left.88^{\circ} 46.5^{\prime} \mathrm{E}\right)$, Rajshahi district. The charland is more or less continuous throughout this part of the river, and at some places cut by small water areas and a few channels. The distance between Premtali to Sardah is approximately 60 kilometers.

The main bird observation sites were three: (i) Premtali, covering an area of 2-3 km EastWest and approximately $1 \mathrm{~km}$ inside the char; (ii) Rajshahi City, covering approximately $10.2 \mathrm{~km}$ long East-West, and around 2-4 km inside the char; and (iii) Sardah Cadet College covering approximately $2 \mathrm{~km}$ East-West and $0.5 \mathrm{~km}$ inside the char. During the survey the water areas, grasslands, and bushes were observed carefully. However, some birds were found in the dry sandy and bare soils by the side of small grasses.

Bird watching in the Padma charland was started since 2008 and still continuing. For nearly 2 years the bird watching was confined at 3-4 sites of the Rajshahi City area such as, Central Park, Boro Kuthi, Alupatti, Talaimari Shaheed Minar \& Kazla. After that period the total study area between Premtali to Sardah (from west to east approximately 60 kilometers) was extensively surveyed to record the avifauna.

Habitats of the charland: Habitats of the study area were categorized as water area and charland. The charland includes four types of habitat, such as, grass land, sandy area, agricultural land and open wood land (plantation areas). The characteristics of the soil of the habitats are different, i.e., sandy, alluvial, clay and sandy-alluvium mixed. Accordingly flora and fauna of these habitats are differently composed.

Bird watching schedule: The study area was extensively surveyed each year from autumn (October) to summer (June). Most regular bird watching activity was in the Rajshahi City area, at least once in a fortnight. However, at the points from Kazla to Talaimari Shaheed Minar the bird observation was carried once in a week, and at the distant sites like Premtali, and Sardah it was 2-3 times in a year from December to early May. Bird watching at the Padma char was mostly started before sun rise, and spent more or less 3-4 hours. During October-June occasional survey was carried at the late afternoon till sunset, for about 2 hours. 
Data Recording: Digital still camera (Nikon D3000 DSLR, $300 \mathrm{~mm}$ Nikor lens) and video camera (Sony DCR SR68) were used for recording data of birds of the charland. A binocular was also used to observe the birds.

Identification of birds: The birds those were photographed were identified in the Department of Zoology, University of Rajshahi consulting the published literature, viz., Baker (1922-1930), Ali (2002), Ali and Ripley (1968-1972), Khan (2008), Siddiquee et al. (2008), Halder (2010) and the Internet (Birdlife International).

During the study period total 141 species of birds were identified from the charland of the Padma river (Reza et al., 2014), of which $58(41.43 \%)$ are migratory to Bangladesh, and the rest $\mathbf{8 3}(58.86 \%)$ are resident of this country. Among these migratory species, 47 $(81.03 \%)$ are water birds and $11(18.96 \%)$ are small passerine species (Table 1$)$.

Total number of winter visitors were recorded as 53 (91.38\%) and among them eight (8) are reported to be resident locally. Two species of water birds, the Tufted Pochard (Aythya fuligula) and the Asian Dowitcher (Limnodromus semipalmatus) are listed as Near Threatened species (IUCN, 2008). There are six (5) species of rare winter visitors and more five (5) species are known as uncommon winter visitors of Bangladesh.

Other than the winter visitors, there found four (4) species of summer visitors $(6.98 \%)$ and two (2) species of passage migrants (3.45\%). Among the summer visitors two (2) species are reported to often reside in Bangladesh. The Grey Bushchat (Saxicola ferrus) is normally a winter visitor, but sometimes they do not pass the whole season, spend few days during the journey within Bangladesh territory as a passage migrant. During the present study this bird could not recorded each year from the Padma charland.

However, flocks of ducks in the main channel were seen from distance, but absence of proper instruments good photographs could not be taken from which the species could be identified. Bird watcher James Pender reported the presence of Teal, Wigeon, Garganey, Shovelar, Common Shelduck and Mallard in the charlands of the Padma river (personal communication). Because of social risks to pass through the tall grassland, the bird watcher team could not reach near the main channel.

Among the total bird species of Bangladesh, 171 species are migratory, of which 160 are winter visitors, six (6) are summer visitors and 10 are passage migrants or spring visitors (Siddiquee et al., 2008). Migratory birds are excellent indicators of the environmental state of a region. The migratory birds play important roles in pest control (insects and rodents) of the croplands and increase the nutrients of the soil by dropping faecal materials, disperse seeds and pollinates.

The Padma charland is totally a virgin area for wildlife study and documentation. The present study is providing a list of the migratory birds of this habitat for the first time. 
Table 1. Check list of migratory birds of the Padma charland (Godagari to Charghat, Rajshahi )

\begin{tabular}{|c|c|c|c|c|c|}
\hline Order/Family & Scientific Name & English Name & Bangla Name & Habitat & $\begin{array}{c}\text { Status in } \\
\text { Bangladesh } \\
\text { (IUCN, 2008) }\end{array}$ \\
\hline \multirow[t]{5}{*}{$\begin{array}{l}\text { Anseriformes } \\
\text { Anatidae }\end{array}$} & $\begin{array}{l}\text { Dendrocygna } \\
\text { bicolor Vieillot, } \\
1816)\end{array}$ & $\begin{array}{l}\text { Large } \\
\text { Whistling } \\
\text { Duck }\end{array}$ & Boro Sarali & $\begin{array}{l}\text { Water } \\
\text { area }\end{array}$ & $\begin{array}{l}\text { Common Winter } \\
\text { Visitor }\end{array}$ \\
\hline & $\begin{array}{l}\text { Aythya fuligula } \\
\text { (Linnaeus, 1758) }\end{array}$ & $\begin{array}{l}\text { Tufted } \\
\text { Pochard }\end{array}$ & Tiki Hans & $\begin{array}{l}\text { Water } \\
\text { area }\end{array}$ & $\begin{array}{l}\text { Common Winter } \\
\text { Visitor }\end{array}$ \\
\hline & $\begin{array}{l}\text { Aythya nyroca } \\
\text { (Guldenstadt, } \\
\text { 1770) }\end{array}$ & $\begin{array}{l}\text { Ferruginous } \\
\text { Duck }\end{array}$ & $\begin{array}{l}\text { Morcherong } \\
\text { Bhuti Hans }\end{array}$ & $\begin{array}{l}\text { Water } \\
\text { area }\end{array}$ & $\begin{array}{l}\text { Common Winter } \\
\text { Visitor (Near } \\
\text { Threatened) }\end{array}$ \\
\hline & $\begin{array}{l}\text { Tadorna } \\
\text { ferruginea } \\
\text { (Pallas, 1964) }\end{array}$ & $\begin{array}{l}\text { Brahminy } \\
\text { Shelduck }\end{array}$ & $\begin{array}{l}\text { Khoira } \\
\text { Chokachoki }\end{array}$ & $\begin{array}{l}\text { Water } \\
\text { area }\end{array}$ & $\begin{array}{l}\text { Common Winter } \\
\text { Visitor }\end{array}$ \\
\hline & $\begin{array}{l}\text { Anas } \\
\text { poecilorhyncha } \\
\text { Forster, } 1781\end{array}$ & $\begin{array}{l}\text { Indian Spot-bi } \\
\text { Duck }\end{array}$ & $\begin{array}{l}\text { Deshi Mete } \\
\text { Hans }\end{array}$ & $\begin{array}{l}\text { Water } \\
\text { area }\end{array}$ & $\begin{array}{l}\text { Common Winter } \\
\text { Visitor } \\
\text { / Resident }\end{array}$ \\
\hline Coraciiformes & Merops & Blue tailed & Neel-lej & Terrestr- & Summer Visitor/ \\
\hline Meropidae & $\begin{array}{l}\text { philippinus } \\
\text { (Linnaeus, 1766) }\end{array}$ & Bee-eater & Suichora & $\begin{array}{l}\text { ial (aerial } \\
\text { eeding) }\end{array}$ & Resident \\
\hline \multirow[t]{2}{*}{$\begin{array}{l}\text { Cuculiformes } \\
\text { Cuculidae }\end{array}$} & $\begin{array}{l}\text { Clamator } \\
\text { jacobinus } \\
\text { (Boddaert, 1783) }\end{array}$ & $\begin{array}{l}\text { Pied-crested } \\
\text { Cuckoo }\end{array}$ & Pakra Papia & $\begin{array}{l}\text { Terrestr- } \\
\text { ial }\end{array}$ & Summer Visitor \\
\hline & $\begin{array}{l}\text { Hierococcyx } \\
\text { varius (Vahl, } \\
\text { 1797) }\end{array}$ & $\begin{array}{l}\text { Common } \\
\text { Hawk } \\
\text { Cuckoo }\end{array}$ & $\begin{array}{l}\text { Pati } \\
\text { Chokhgelo }\end{array}$ & $\begin{array}{l}\text { Terrestr- } \\
\text { ial }\end{array}$ & Summer Visitor \\
\hline \multirow{11}{*}{$\begin{array}{l}\text { Ciconiiformes } \\
\text { colopacidae }\end{array}$} & Tringa glareola & Wood & Tila & Water & Common Winter \\
\hline & Linnaeus, 1758 & Sandpiper & Chapakhi & & Visitor \\
\hline & $\begin{array}{l}\text { Tringa ochropus } \\
\text { Linnaeus, } 1758\end{array}$ & $\begin{array}{l}\text { Green } \\
\text { Sandpiper }\end{array}$ & Sabuj Batan & $\begin{array}{l}\text { Water } \\
\text { area }\end{array}$ & $\begin{array}{l}\text { Uncommon } \\
\text { Winter Visitor }\end{array}$ \\
\hline & $\begin{array}{l}\text { Tringa stagnatilis } \\
\text { (Bechstein, 1803) }\end{array}$ & $\begin{array}{l}\text { Marsh } \\
\text { Sandpiper }\end{array}$ & Beel Batan & $\begin{array}{l}\text { Water } \\
\text { area }\end{array}$ & $\begin{array}{l}\text { Uncommon } \\
\text { Winter Visitor }\end{array}$ \\
\hline & $\begin{array}{l}\text { Tringa nebularia } \\
\text { (Gunnerus, 1767) }\end{array}$ & $\begin{array}{l}\text { Common } \\
\text { Greenshank }\end{array}$ & Pati Sabujpa & $\begin{array}{l}\text { Water } \\
\text { area }\end{array}$ & $\begin{array}{l}\text { Common Winter } \\
\text { Visitor }\end{array}$ \\
\hline & $\begin{array}{l}\text { Actitis } \\
\text { hypoleucos } \\
\text { Linnaeus, } 1758\end{array}$ & $\begin{array}{l}\text { Common } \\
\text { Sandpiper }\end{array}$ & Pati Batan & $\begin{array}{l}\text { Water } \\
\text { area }\end{array}$ & $\begin{array}{l}\text { Common Winter } \\
\text { Visitor }\end{array}$ \\
\hline & $\begin{array}{l}\text { Calidris alpina } \\
\text { (Linnaeus, 1758) }\end{array}$ & Dunlin & Dunlin & $\begin{array}{l}\text { Water } \\
\text { area }\end{array}$ & $\begin{array}{l}\text { Rare Winter } \\
\text { Visitor }\end{array}$ \\
\hline & $\begin{array}{l}\text { Calidris alba } \\
\text { (Pallas, 1764) }\end{array}$ & Sanderling & Sanderling & $\begin{array}{l}\text { Water } \\
\text { area }\end{array}$ & $\begin{array}{l}\text { Uncommon } \\
\text { Winter Visitor }\end{array}$ \\
\hline & $\begin{array}{l}\text { Calidris } \\
\text { temminckii } \\
\text { (Leisler, 1812) }\end{array}$ & $\begin{array}{l}\text { Temminck's } \\
\text { Stint }\end{array}$ & $\begin{array}{l}\text { Temminker } \\
\text { Chaha }\end{array}$ & $\begin{array}{l}\text { Water } \\
\text { area }\end{array}$ & $\begin{array}{l}\text { Common Winter } \\
\text { Visitor }\end{array}$ \\
\hline & $\begin{array}{l}\text { Calidris minuta } \\
\text { (Leisler, 1812) }\end{array}$ & Little Stint & $\begin{array}{l}\text { Chhoto } \\
\text { Chaha }\end{array}$ & $\begin{array}{l}\text { Water } \\
\text { area }\end{array}$ & $\begin{array}{l}\text { Common Winter } \\
\text { Visitor }\end{array}$ \\
\hline & $\begin{array}{l}\text { Calidris ruficolis } \\
\text { (Pallas, 1776) }\end{array}$ & $\begin{array}{l}\text { Red-necked } \\
\text { Stint }\end{array}$ & $\begin{array}{l}\text { Lalghar } \\
\text { Chaha }\end{array}$ & $\begin{array}{l}\text { Water } \\
\text { area }\end{array}$ & $\begin{array}{l}\text { Common Winter } \\
\text { Visitor }\end{array}$ \\
\hline
\end{tabular}




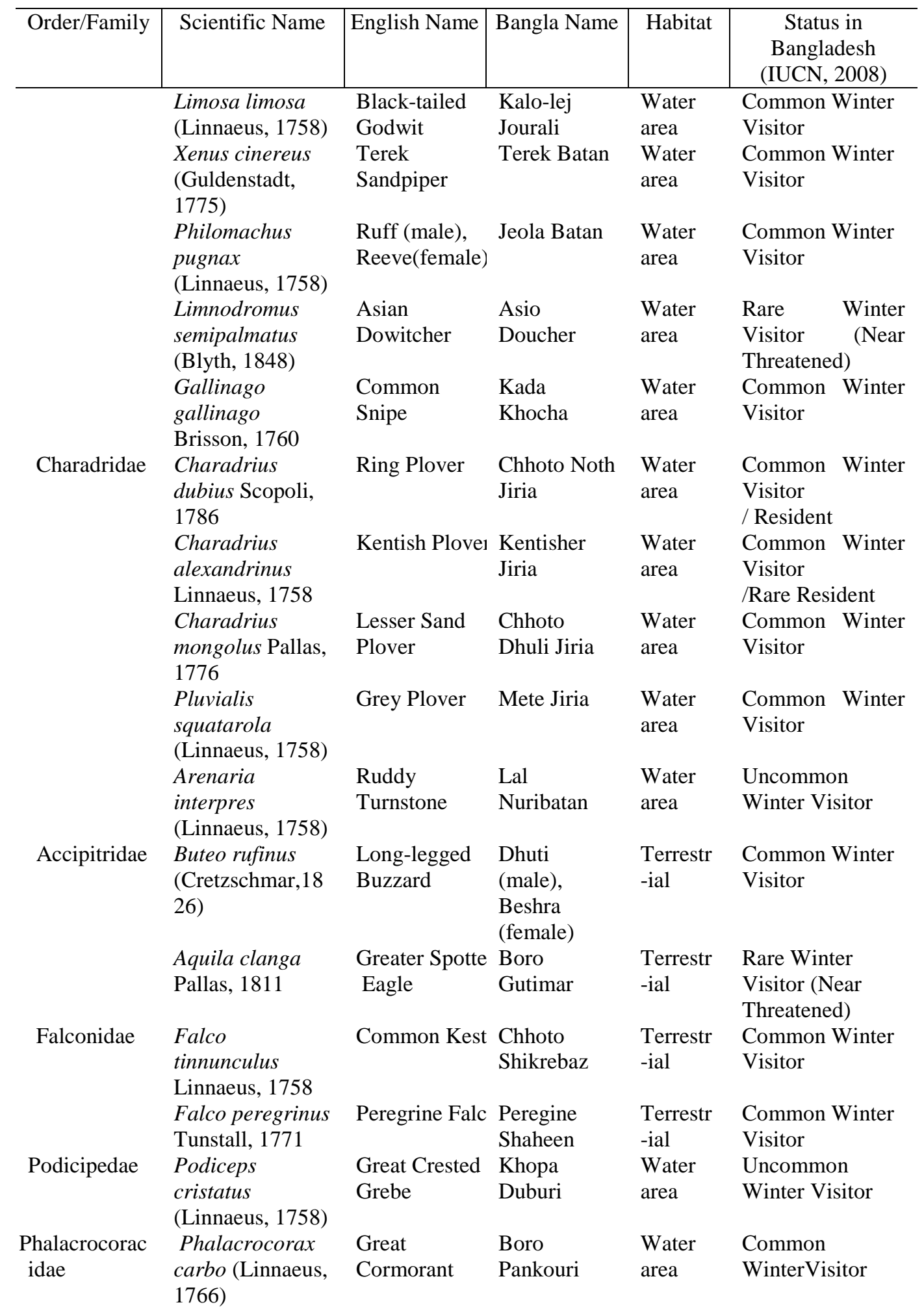




\begin{tabular}{|c|c|c|c|c|c|}
\hline Order/Family & Scientific Name & English Name & Bangla Name & Habitat & $\begin{array}{c}\text { Status in } \\
\text { Bangladesh } \\
(\mathrm{IUCN}, 2008) \\
\end{array}$ \\
\hline Ardeidae & $\begin{array}{l}\text { Ardea cinerea } \\
\text { Linnaeus, } 1758\end{array}$ & Grey Heron & $\begin{array}{l}\text { Dhushar } \\
\text { Bok }\end{array}$ & $\begin{array}{l}\text { Water } \\
\text { area }\end{array}$ & $\begin{array}{l}\text { Common Winter } \\
\text { Visitor/ Resident }\end{array}$ \\
\hline \multirow[t]{3}{*}{$\begin{array}{l}\text { Passeriformes } \\
\text { Lanidae }\end{array}$} & $\begin{array}{l}\text { Lanius cristatus } \\
\text { Linnaeus, } 1758 \\
\text { Terpsiphone } \\
\text { paradise } \\
\text { (Latham, 1758) }\end{array}$ & $\begin{array}{l}\text { Brown } \\
\text { Shrike } \\
\text { Paradise } \\
\text { Flycatcher }\end{array}$ & $\begin{array}{l}\text { Badami } \\
\text { Koshai Pakhi } \\
\text { Shah Bulbul }\end{array}$ & $\begin{array}{l}\text { Terrestr- } \\
\text { ial } \\
\text { Terrestr- } \\
\text { ial }\end{array}$ & $\begin{array}{l}\text { Common Winter } \\
\text { Visitor } \\
\text { Summer Visitor } \\
\text { /Resident }\end{array}$ \\
\hline & $\begin{array}{l}\text { Pericrocotus } \\
\text { divaricatus } \\
\text { (Raffles, 1822) }\end{array}$ & Ashy Minivet & $\begin{array}{l}\text { Dhushar Sat } \\
\text { Saheli }\end{array}$ & $\begin{array}{l}\text { Terrestr- } \\
\text { ial }\end{array}$ & $\begin{array}{l}\text { Common Winter } \\
\text { Visitor }\end{array}$ \\
\hline & $\begin{array}{l}\text { Dicrurus } \\
\text { leucophaeus } \\
\text { Vieillot, } 1817\end{array}$ & $\begin{array}{l}\text { Ashy } \\
\text { Drongo }\end{array}$ & $\begin{array}{l}\text { Dhusar } \\
\text { Fingey }\end{array}$ & $\begin{array}{l}\text { Terrestr- } \\
\text { ial }\end{array}$ & $\begin{array}{l}\text { Common Winter } \\
\text { Visitor }\end{array}$ \\
\hline \multirow[t]{8}{*}{ Muscicapidae } & $\begin{array}{l}\text { Muscicapa } \\
\text { dauurica Pallas, } \\
1811\end{array}$ & $\begin{array}{l}\text { Asian- } \\
\text { brown } \\
\text { Flycatcher }\end{array}$ & $\begin{array}{l}\text { Badami } \\
\text { Chatak }\end{array}$ & $\begin{array}{l}\text { Terrestr- } \\
\text { ial }\end{array}$ & Passage Migrant \\
\hline & $\begin{array}{l}\text { Eumyias } \\
\text { thalassina } \\
\text { Swainson, } 1838\end{array}$ & $\begin{array}{l}\text { Verditer } \\
\text { Flycatcher }\end{array}$ & $\begin{array}{l}\text { Firoza } \\
\text { Chotok }\end{array}$ & $\begin{array}{l}\text { Terrestr- } \\
\text { ial }\end{array}$ & $\begin{array}{l}\text { Common Winter } \\
\text { Visitor }\end{array}$ \\
\hline & $\begin{array}{l}\text { Saxicola ferrues } \\
\text { (Gray, 1846) }\end{array}$ & $\begin{array}{l}\text { Grey } \\
\text { Bushchat }\end{array}$ & $\begin{array}{l}\text { Mete Jhar } \\
\text { Fidda }\end{array}$ & $\begin{array}{l}\text { Terrestr- } \\
\text { ial }\end{array}$ & $\begin{array}{l}\text { Winter Visitor } \\
\text { / Passage Migrant }\end{array}$ \\
\hline & Saxicola insignis & Hodgson's & Dholagola & Terrestr- & Common Winter \\
\hline & Gray, 1846 & Bushchat & Jhar Fidda & ial & Visitor \\
\hline & $\begin{array}{l}\text { Saxicola leucura } \\
\text { (Blyth, 1847) }\end{array}$ & $\begin{array}{l}\text { White-tailed } \\
\text { Stonechat }\end{array}$ & $\begin{array}{l}\text { Sada-lej } \\
\text { Fidda }\end{array}$ & $\begin{array}{l}\text { Terrestr- } \\
\text { ial }\end{array}$ & $\begin{array}{l}\text { Common Winter } \\
\text { Visitor }\end{array}$ \\
\hline & $\begin{array}{l}\text { Saxicola } \\
\text { toquatas } \\
\text { (Linnaeus, 1766) }\end{array}$ & $\begin{array}{l}\text { Eurasian } \\
\text { Stoechat/ } \\
\text { Common } \\
\text { Stonechat }\end{array}$ & $\begin{array}{l}\text { Pati Shila } \\
\text { Fidda }\end{array}$ & $\begin{array}{l}\text { Terrestr- } \\
\text { ial }\end{array}$ & $\begin{array}{l}\text { Common Winter } \\
\text { Visitor }\end{array}$ \\
\hline & $\begin{array}{l}\text { Luscinia svecica } \\
\text { Linnaeus, } 1758\end{array}$ & Bluethroat & $\begin{array}{l}\text { Neelgola } \\
\text { Fidda }\end{array}$ & $\begin{array}{l}\text { Terrestr- } \\
\text { ial }\end{array}$ & $\begin{array}{l}\text { Common Winter } \\
\text { Visitor }\end{array}$ \\
\hline Hirundidae & $\begin{array}{l}\text { Riparia riparia } \\
\text { (Linnaeus, 1758) }\end{array}$ & Sand Martin & Balu Nakuti & $\begin{array}{l}\text { Water } \\
\text { area }\end{array}$ & $\begin{array}{l}\text { Common Winter } \\
\text { Visitor }\end{array}$ \\
\hline \multirow[t]{2}{*}{ Sylviidae } & $\begin{array}{l}\text { Acrocephalus } \\
\text { dumetorum } \\
\text { Blyth, } 1849\end{array}$ & $\begin{array}{l}\text { Brown Back } \\
\text { Reed } \\
\text { Warbler }\end{array}$ & $\begin{array}{l}\text { Badami Pith } \\
\text { Nolphutki }\end{array}$ & $\begin{array}{l}\text { Near } \\
\text { Water } \\
\text { area }\end{array}$ & $\begin{array}{l}\text { Common Winter } \\
\text { Visitor }\end{array}$ \\
\hline & $\begin{array}{l}\text { Sylvia curruca } \\
\text { (Linnaeus, 1758) }\end{array}$ & $\begin{array}{l}\text { Lesser } \\
\text { Whitethroat }\end{array}$ & $\begin{array}{l}\text { Chhoto } \\
\text { Dholagola }\end{array}$ & $\begin{array}{l}\text { Terrestr- } \\
\text { ial }\end{array}$ & $\begin{array}{l}\text { Rare } \\
\text { Visitor }\end{array}$ \\
\hline \multirow[t]{3}{*}{ Passeridae } & $\begin{array}{l}\text { Motacilla } \\
\text { citriola Pallas, } \\
1776\end{array}$ & $\begin{array}{l}\text { Citrine } \\
\text { Wagtail }\end{array}$ & $\begin{array}{l}\text { Holde-matha } \\
\text { Khonjon }\end{array}$ & $\begin{array}{l}\text { Near } \\
\text { Water } \\
\text { area }\end{array}$ & $\begin{array}{l}\text { Common Winter } \\
\text { Visitor }\end{array}$ \\
\hline & $\begin{array}{l}\text { Motacilla alba } \\
\text { alba Linnaeus, } \\
1758\end{array}$ & $\begin{array}{l}\text { White } \\
\text { Wagtail }\end{array}$ & $\begin{array}{l}\text { Sada } \\
\text { Khonjon }\end{array}$ & $\begin{array}{l}\text { Near } \\
\text { Water } \\
\text { area }\end{array}$ & $\begin{array}{l}\text { Common Winter } \\
\text { Visitor }\end{array}$ \\
\hline & $\begin{array}{l}\text { M. a. leucopsis } \\
\text { (Linnaeus, 1758) }\end{array}$ & $\begin{array}{l}\text { White } \\
\text { Wagtail } \\
\text { (sub-sp.) }\end{array}$ & $\begin{array}{l}\text { Sada } \\
\text { Khonjon } \\
\text { (sub-sp.) }\end{array}$ & $\begin{array}{l}\text { Near } \\
\text { Water } \\
\text { area }\end{array}$ & $\begin{array}{l}\text { Common Winter } \\
\text { Visitor }\end{array}$ \\
\hline
\end{tabular}




\begin{tabular}{|c|c|c|c|c|c|}
\hline Order/Family & Scientific Name & English Name & Bangla Name & Habitat & $\begin{array}{c}\text { Status in } \\
\text { Bangladesh } \\
(\mathrm{IUCN}, 2008) \\
\end{array}$ \\
\hline & $\begin{array}{l}\text { M. a. personata } \\
\text { (Linnaeus, 1758) }\end{array}$ & $\begin{array}{l}\text { White } \\
\text { Wagtail } \\
\text { (sub-sp.) }\end{array}$ & $\begin{array}{l}\text { Sada } \\
\text { Khonjon } \\
\text { (sub-sp.) }\end{array}$ & $\begin{array}{l}\text { Near } \\
\text { Water } \\
\text { area }\end{array}$ & $\begin{array}{l}\text { Common Winter } \\
\text { Visitor }\end{array}$ \\
\hline & Motacilla & Grey & Dhusar & Near & Common Winter \\
\hline & $\begin{array}{l}\text { cinerea Tunstall, } \\
1771\end{array}$ & Wagtail & Khonjon & $\begin{array}{l}\text { Water } \\
\text { area }\end{array}$ & Visitor \\
\hline & $\begin{array}{l}\text { Anthus richardi } \\
\text { Vieillot, } 1818\end{array}$ & Richard's Pipi & $\begin{array}{l}\text { Richarder } \\
\text { Tulika }\end{array}$ & $\begin{array}{l}\text { Terrestr- } \\
\text { ial }\end{array}$ & $\begin{array}{l}\text { Common Winter } \\
\text { Visitor }\end{array}$ \\
\hline & Anthus similis & Brown Rock & Lombathot & Terrestr- & Rare Winter \\
\hline & Jerdon, 1840 & Pipit & Tulika & ial & Visitor \\
\hline & $\begin{array}{l}\text { Anthus } \\
\text { campestris } \\
\text { (Linnaeus, 1758) }\end{array}$ & Tawny Pipit & $\begin{array}{l}\text { Tamatey } \\
\text { Tulika }\end{array}$ & $\begin{array}{l}\text { Terrestr- } \\
\text { ial }\end{array}$ & Passage Migrant \\
\hline & $\begin{array}{l}\text { Emberiza pusilla } \\
\text { Pallas, } 1776\end{array}$ & $\begin{array}{l}\text { Little } \\
\text { Bunting }\end{array}$ & $\begin{array}{l}\text { Khude } \\
\text { Chotok }\end{array}$ & $\begin{array}{l}\text { Terrestr- } \\
\text { ial }\end{array}$ & $\begin{array}{l}\text { Common Winter } \\
\text { Visitor }\end{array}$ \\
\hline
\end{tabular}

Acknowledgements: The authors are thankful to the University Grant Commission and the University of Rajshahi for providing fund for the project entitled "Bird Diversity in Rajshahi District' which partially covered the cost of this lengthy work. The assistance provided during the bird watching and preparation of this manuscript by Late Professor Dr Mannan, Department of Zoology, University of Rajshahi, are cordially acknowledged. Assistance of the members of the Bird Watching Team of the Department of Zoology especially, Dr Md Ariful Hasan, Associate Professor, Al-Amin, Md Shahinoor Rahman and Sheikh Md Atiqur Rahman is thankfully acknowledged.

\section{REFERENCES}

Ali, S. 2002. The Book of Indian Birds $\left(13^{\text {th }}\right.$ edn.). Bombay Natural History Society, Oxford University Press, Oxford, New York.

Ali, S. and Ripley, S.D. 1968-1973. Handbook of the Birds of India and Pakistan, (9 vols.), Oxford University Press, Bombay, London, New York.

Ali, A.A., Sultana, S. and Parween, S. 2013. Fishes of Rajshahi region deposited in the Zoology Museum (in Bangla). Prabal 28:32-41.

Baker E.C. S. 1922-1930. Fauna of British India, Birds (Vols. 1-8). Taylor and Francis, London.

Claassen, A.H. 2004. Abundance, Distribution and Reproduction Success of Sandbar Nesting Birds Below the Yali Falls Hydropower Dam on the Sesan River, Northeastern Cambodia. World Wide Fund (WWF) for Nature, Phnom Penh, Cambodia.

Halder, R. R. 2010. A Photographic Guide to Birds of Bangladesh. Baikal Teal Publication.

Islam, M.S. and Hossain, M.A. 1983. An account of the fishes of the Padma river near Rajshahi. Rajshahi Fisheries Bulletin 1(2):1-31.

IUCN-Bangladesh, 2008. Red list of threatened birds of Bangladesh. The World Conservation Union, Dhaka.

Khan, M.A.R. 1973. Checklist of birds of Rajshahi with their ecological groupings. Rajshahi University Studies 5:200-213.

Khan, R. 2008. Birds of Bangladesh (in Bangla). Bangla Academy, Dhaka. 
Rahman, M.A. and Rahman, M.M. 2012. Char Formation Process and Livelihood Characteristics of Char Dwellers of Alluvial River in Bangladesh. ICSE 6 Paris- August 27-31, 2012.

Reza, A.M. Saleh, Al-Amin, M., Rahman, S.M.A., Rahman, M. S., Hasan, M. A. and Parween, S. 2014. A checklist of waterbirds of the Padma river charland (Godagari to Charghat), Rajshahi, Bangladesh. univ. j.zool., Rajshahi university 33 (in press).

Sarker M. H., Iffat H. and Mustafa A. 2003. Rivers, chars and char people of Bangladesh. International Journal of River Basin Management, IAHR, 1 (1): 61-80.

Siddique, K. U., Islam, M. A., Kabir, S. M. H., Ahmed, M., A., Rahman, A.T. A., Rahman, A. K. A., Haque, E. U., Ahmed, Z. U., Begum, Z. N. T., Hassan, M. A., Khondker, M. and Rahman, M. M. (eds.) 2008. Encyclopedia of Flora and Fauna of Bangladesh. Birds (Vol. 26), Asiatic Society of Bangladesh. 